A N N A L E S Annales de Bretagne et des Pays de l'Ouest

\title{
Liens de sang, liens de pouvoir
}

\section{Mathieu Pichard}

\section{(2) OpenEdition}

Journals

Édition électronique

URL : http://journals.openedition.org/abpo/2530

DOI : $10.4000 /$ abpo. 2530

ISBN : 978-2-7535-2236-7

ISSN : 2108-6443

Éditeur

Presses universitaires de Rennes

Édition imprimée

Date de publication : 31 décembre 2012

Pagination : 153-154

ISBN : 978-2-7535-2234-3

ISSN : 0399-0826

Référence électronique

Mathieu Pichard, "Liens de sang, liens de pouvoir », Annales de Bretagne et des Pays de l'Ouest [En ligne], 119-4 | 2012, mis en ligne le 31 décembre 2012, consulté le 09 juillet 2020. URL : http:// journals.openedition.org/abpo/2530

Ce document a été généré automatiquement le 9 juillet 2020

(c) Presses universitaires de Rennes 


\title{
Liens de sang, liens de pouvoir
}

\author{
Mathieu Pichard
}

\section{RÉFÉRENCE}

Coste, Laurent (dir.), Liens de sang, liens de pouvoir. Les élites dirigeantes urbaines en Europe occidentale et dans les colonies européennes (fin $\mathrm{xv}^{\mathrm{e}}$-fin $\mathrm{xix}^{\mathrm{e}}$ siècle), Rennes, Presses universitaires de Rennes, 2010, 349 p., ISBN 978-2-7535-1042-2, $18 €$.

1 Cet ouvrage, publié sous la direction de Laurent Coste, propose une approche multiscalaire des élites dirigeantes urbaines et de leurs liens de parenté entre la fin du $\mathrm{xv}^{\mathrm{e}}$ et la fin $\mathrm{du} \mathrm{xix}^{\mathrm{e}}$ siècle. Les textes qu'il réunit constituent assurément une contribution importante et enrichissante à l'œuvre de renouvellement historiographique entreprise en histoire urbaine depuis la fin des années 1990, dont l'originalité est de raisonner au niveau de l'espace atlantique et de ses prolongements coloniaux. Ils présentent, sous les plumes d'historiens français, anglais, espagnols et portugais, seize approches de l'engagement politique municipal analysé à la lumière des lignages, liens familiaux et parentés qui unissent les membres des institutions urbaines. Partant d'une analyse comparative en termes d'espaces étudiés (Guy Saupin, Jean-Philippe Priotti, Nuno Gonçalo Monteiro et Vicente Montojo Montojo s'intéressent aux villes maritimes au sein d'un ou plusieurs espaces "nationaux»), l'échelle d'analyse s'affine progressivement vers les espaces régionaux (Philippe Jarnoux, Stéphane Durand, Agathe Leyssens et Jean-Paul Jourdan proposent de relativiser l'opposition entre villes portuaires et intérieures au sein d'une même province: Bretagne, Bas-Languedoc, Flandres et Aquitaine). Dans une troisième partie, l'espace urbain proprement dit est privilégié par les études d'Olivia Carpi sur Amiens, de Béatrice Beaumier sur Tours, de Christine Lamarre sur Dijon et de Jean-Pierre Poussou sur une dizaine de villes du département du Lot-et-Garonne. Enfin, la dernière partie se concentre sur quatre destins familiaux ou individuels sous les plumes d'Alexandre Fernandez, Dominique Goncalvès, Yann Lignereux et Perry Gauci. 
2 Au gré des chapitres successifs, le clivage social et professionnel évoqué parfois dans l'historiographie entre les corps de ville des cités maritimes et ceux des villes continentales est nuancé ou à l'inverse conforté. En Bretagne d'abord, où P. Jarnoux constate que la hiérarchie des pouvoirs au sein des groupes municipaux est dans les deux cas "nettement favorable aux officiers royaux" (p.119), en particulier aux officiers de justice, sans que l'on puisse distinguer de fracture nette entre les petites villes, majoritairement fluvio-maritimes, et les villes continentales, fluviales ou non. P. Jarnoux enrichit la connaissance du réseau municipal et officier de l'Ouest français (connaissance déjà renforcée depuis la publication de l'ouvrage collectif dirigé par G. Saupin, Histoire sociale du politique, Rennes, Presses universitaires de Rennes, 2010 compte-rendu dans $A B P O, 2011 / 2$, p. 180-183) en établissant une corrélation entre la rareté de l'office breton (moins de 500 personnes en 1660) et la grande proportion de titulaires nobles qui président les séances des municipalités et surveillent la régularité des procédures électorales. Il insiste donc sur la distance qui sépare ce monde de l'office des élites municipales, distance à la fois sociale, professionnelle et pratique que renforce le rythme quotidien de l'action des corps de ville bretons (les officiers organisent la police urbaine, ont préséance lors des cérémonies publiques et reçoivent prioritairement les courriers du roi). Les réalités politiques des villes littorales ou portuaires, comme Nantes, relativisent cette distance dans la mesure où les réussites marchandes et maritimes permettent des succès économiques qui catalysent la fusion et le renouvellement des élites selon des logiques socio-professionnelles variables et spécifiques. Dans les Flandres, A. Leyssens montre que les modalités d'accès à l'échevinage dépendent moins de l'opposition géographique littoral/intérieur que du poids plus ou moins grand de l'intervention du roi (qui s'exprime par l'intervention du commissaire royal dans la nomination des nouveaux membres du magistrat). Elle établit ainsi que " les villes dont l'intégration au royaume des lys est la plus ancienne et où l'économie est la plus atone subissent davantage la férule du pouvoir royal et connaissent une fermeture plus marquée des rangs scabinaux aux nouveaux venus " (p. 169). À l'inverse - mais pour une période bien plus tardive - l'étude de J.-P. Jourdan établit une distinction nette entre les villes maritimes de l'Aquitaine au xix ${ }^{\mathrm{e}}$ siècle (Bordeaux et Bayonne) et les villes de l'intérieur (Périgueux, Agen ou Pau) qui se perpétue avant et après 1831, c'est-à-dire à partir de l'élection des conseils municipaux au suffrage restreint. Dans les premières, il observe la mainmise des négociants sur les municipalités, "la composition sociale des conseils municipaux reflétant alors leur fonction dominante, et donc la nature de leurs élites respectives" (p.182). Tout en mettant en lumière des éléments récurrents de continuité dans la formation des lignages municipaux, l'ouvrage contribue à souligner les particularités des situations locales, dont G. Saupin montre bien qu'elles nuancent considérablement l'idée d'une structuration «atlantique » uniforme des sociologies urbaines anglaise et française. S. Durand observe également des variations locales dans le contexte général de la structuration du bas Languedoc en consulats, au xviiie siècle. À Mèze, l'autorité seigneuriale, en l'occurrence celle de l'évêque d'Agde, sur la désignation des consuls est formelle et ne s'exprime que par simple validation d'un juge tandis que la petite taille de la ville et l'absence de fonction administrative importante minorent le rôle des officiers et laissent le champ libre aux négociants et aux bourgeois. Quant aux situations des villes continentales de Tours, Amiens et Dijon, il apparaît que l'émergence ou non de dynasties municipales et le renouvellement plus ou moins fort des élites urbaines, lorsque les sources permettent de le dire, sont intimement liées aux 
configurations socio-professionnelles locales et aux rythmes chronologiques différents de la réglementation royale. L'un des intérêts du livre est donc de montrer, parallèlement aux mécanismes de reproduction lignagère des familles (Y. Lignereux insiste en particulier sur les stratégies matrimoniales des Villeroy), la variabilité des situations locales en fonction de paramètres clairement identifiés: relations avec le pouvoir, modalités d'accès au pouvoir ou encore prétentions dynastiques d'une famille sur le long ou moyen terme.

3 Enfin, les études réunies par L. Coste engagent à une utilisation plus fine du concept de « catégorie sociale» en ne réduisant pas les hommes à des représentants de groupes sociaux appelés par leur inscription sociale à une sorte de partage tacite ou réglementaire du pouvoir. Ainsi, le succès de la famille Ybarra à Bilbao, présenté par A. Fernandez, s'explique essentiellement par la «stratégie de réussite du fondateur de la dynastie » (p. 278) dans un contexte régional et littoral économiquement favorable à partir de la fin du xviiie siècle. Dans un milieu où les alliances matrimoniales remodèlent ou confortent la position socio-professionnelle, le pouvoir dans la ville s'identifie à une forme de paternalisme industriel marqué par une éthique capitaliste qui permet à la famille Ybarra de constituer une oligarchie à partir de la fin du xix ${ }^{\mathrm{e}}$ siècle. D. Goncalvès décrit des phénomènes semblables lorsqu'il présente l'ascension de Diego Peñalver à partir de 1681, date à laquelle la famille s'implante définitivement à La Havane où Diego et ses descendants jouissent d'une charge prestigieuse de trésorier royal. À l'échelle d'un individu, l'étude de P. Gauci présente le planteur créole William Beckford comme un homme dynamique et transatlantique dont l'identité sociale se définit à mesure qu'il développe un réseau de connaissances multiples. Il montre comment Beckford s'est servi des institutions municipales (il devient échevin en 1752 et membre de la City de Londres en 1754) comme d'un tremplin vers la défense, notamment fiscale, du «lobby du sucre » (p.271) jamaïcain dans les hautes sphères parlementaires puis ministérielles. L'ensemble des études réunies dans cet ouvrage montre que la construction d'une demeure luxueuse, l'implication dans la vie paroissiale du quartier, le renouvellement constant d'amitiés marchandes ou aristocratiques ainsi que l'acquisition d'une propriété foncière dans la campagne de la ville sont autant d'étapes qui jalonnent l'intégration du "patricien urbain» aux réseaux de la ville et le développement progressif d'une culture citadine sur laquelle les archives restent parfois silencieuses avant le xviiie siècle. 